\title{
ON THE BOUNDARY-VALUE PROBLEM OF A SPHEROID*
}

\author{
By JOHN G. FIKIORIS (University of Toledo, Toledo, Ohio)**
}

\begin{abstract}
The surface charge density of a charged spheroid is obtained in exact, closed form using a Green's function expansion in spherical coordinates. The possibility is thus established of solving boundary-value problems analytically using coordinates that do not correspond to boundary shapes. The present approach, used previously in numerical solutions of related problems, requires the potential function to be constant in the interior of the conductor. Its advantages from the theoretical standpoint and its further possibilities are discussed.
\end{abstract}

1. Introduction. Solution of boundary-value problems by separation of variables is severely limited by the shape of boundaries. For general shapes, problems in potential theory or electromagnetic scattering are formulated in terms of surface (two-dimensional) singular integral equations [1]. Even then analytical solutions are limited to separable systems which fit the boundaries and in which the kernel function $1 / R=\left[\left(x-x^{\prime}\right)^{2}+\right.$ $\left.\left(y-y^{\prime}\right)^{2}+\left(z-z^{\prime}\right)^{2}\right]^{-1 / 2}$ can be expanded in a series of orthogonal eigenfunctions. If these conditions are not met numerical methods are the only alternative.

It is the purpose of this paper to show that analytical approaches need not be so limited. The method is best explained by considering the electrostatic problem of a charged perfect conductor. Let $S$ denote the closed surface of a perfect conductor and, following Kellogg [1], let points on $S$ be designated by small letters (such as $q, p$ ), while capitals (such as $Q, P$ ) are used for points not on $S$, as shown in Fig. 1. Use of Green's theorem in the region $T$ outside $S$ yields the following equation for the potential function $V(P)$ arising from surface charge densities on $S$ [1, pp. 219 and 223], [2, p. 15]:

$$
\left[\begin{array}{c}
V(P) \\
0
\end{array}\right]=\frac{1}{4 \pi} \int_{s}\left[\frac{1}{R} \frac{\partial V}{\partial n^{\prime}}-V(q) \frac{\partial}{\partial n^{\prime}}\left(\frac{1}{R}\right)\right] d S^{\prime} \quad \text { for } \quad P\left[\begin{array}{l}
\text { in } T \text {, i.e. outside } S, \\
\text { out of } T \text {, i.e. inside } S,
\end{array}\right.
$$

where $R$ is the distance from $q\left(r^{\prime}, \theta^{\prime}, \phi^{\prime}\right)$ to $P(r, \theta, \phi)$. In particular, for a perfect conductor at constant potential on $S$, say $V(q)=1$, the second integral can be evaluated by careful application of the divergence theorem [1, pp. 67-68], giving:

$$
\int_{S} \frac{\partial}{\partial n^{\prime}}\left(\frac{1}{R}\right) d S^{\prime}=-\int_{S} \frac{\partial}{\partial n}\left(\frac{1}{R}\right) d S^{\prime}=\left[\begin{array}{c}
0 \\
4 \pi
\end{array} .\right.
$$

The surface charge density on $S$ is $f(q)=\epsilon_{o}\left(\partial V / \partial n^{\prime}\right)$. These two results substituted in the preceding equation yield:

$$
\left.\begin{array}{c}
V(P) \\
1
\end{array}\right]=\frac{1}{4 \pi \epsilon_{o}} \int_{S}[f(q) / R] d S^{\prime} \text { for } P\left[\begin{array}{l}
\text { outside } S \\
\text { inside } S
\end{array} .\right.
$$

* Received January 1, 1972; revised version received March 18, 1972.

** Now at the National Technical University of Athens, Athens, Greece. 
As defined in (1), the potential function $V(P)$ is continuous throughout space. It is analytic (possessing continuous derivatives of all orders in $r, \theta, \phi)$ at all points $P$ not on $S$ (a property shared by solutions of elliptic differential equations) and non-analytic on $S$ [1]. If $P$ is allowed to approach a point $p$ on $S$, a singular integral equation results for $f(q)$ under certain restrictions on the smoothness of $S$. Existence of a unique solution for $f(q)$ is a classical problem treated authoritatively in [1]. With the origin $O$ of a spherical system of coordinates inside $S$ there are always points $P(r, \theta, \phi)$ inside $S$ with $r<r^{\prime}$ for all points $q\left(r^{\prime}, \theta^{\prime}, \phi^{\prime}\right)$ on $S$ (see Fig. 1). Then [3]:

$$
\begin{aligned}
& 1 / R=\sum_{n=0}^{\infty} \sum_{m=0}^{n} \epsilon_{m} \frac{(n-m) !}{(n+m) !} P_{n}^{m}\left(\cos \theta^{\prime}\right) P_{n}^{m}(\cos \theta) \cos m\left(\phi^{\prime}-\phi\right) \frac{r^{n}}{r^{\prime n+1}} \\
& \epsilon_{0}=1, \epsilon_{m}=2 \quad(m>0),
\end{aligned}
$$

where (2) is valid for all points $P$ interior to the circle of radius $r_{m}$ shown in Fig. 1 . Substitution of (2) in (1) for $P$ inside $S$ expresses the constant 1 as a double infinite series expansion in terms of $r^{n}, P_{n}^{m}(\cos \theta)$ and $\cos m \phi, \sin m \phi$ over $0 \leq r \leq r_{m}, 0 \leq \theta \leq \pi$ and $0 \leq \phi \leq 2 \pi$. The orthogonal properties of the angular functions can then be invoked to evaluate the coefficients of the expansion resulting in the set of equations:

$$
\begin{aligned}
4 \pi \epsilon_{o} \delta_{n 0} \delta_{m 0}=\int_{S} \frac{P_{n}\left(\cos \theta^{\prime}\right)}{r^{\prime n+1}} \underset{\sin }{\cos } m \phi^{\prime} f\left(\theta^{\prime}, \phi^{\prime}\right) d S^{\prime} ; \\
n=0,1,2, \cdots ; m=0,1, \cdots, n .
\end{aligned}
$$

Here $\delta_{n 0}=1$ for $n=0, \delta_{n 0}=0$ for $n \neq 0$. Expansion of $1 / R$ in eigenfunctions of Laplace's equation in other coordinate systems provides forms equivalent to (3), which are more suitable for certain boundary shapes.

The method has been used by Smythe $[4,5]$ in electrostatic problems (cylindrical coordinates were used in one case) and, more recently, by Waterman [6,7] in electromagnetic scattering. All these approaches solve equations equivalent to (3) numerically. Here an analytical solution of (3) for $f(q)$ is obtained for the charged spheroid, a shape that does not fit spherical coordinates used in (3). The result for $f(q)$ is the exact, closed form known from separation of variables in spheroidal coordinates and shows that, after all, even in analytical approaches, boundary shape need not exclude a certain coordinate system in which a convenient Green's function expansion is available. Furthermore, it goes one step further than previous numerical results [4-7] in establishing Eq. (3), or its equivalent forms, as a complete formulation of the boundary value problem. Such proof is not yet available, in the same sense that the surface integral equation for $f(q)$ is established as a complete formulation of the problem, subject to precise conditions on smoothness and yielding a unique solution. The analytical results herein justify use of the method, although they do not constitute a general proof of it.

2. The charged prolate spheroid. In prolate spheroidil coordinates $\xi, \eta, \phi[3, \mathrm{pp}$. 1274 and 1284-96] a prolate spheroid of radius $a$ with foci at $x=y=0, z= \pm c$, is defined by

$$
\xi=\xi_{o}=\left(1+a^{2} / c^{2}\right)^{1 / 2} .
$$

When charged to unit potential its surface charge density is easily evaluated in spheroidal coordinates [3]: 


$$
f(\eta)=\epsilon_{o} /\left[a\left(\xi_{o}^{2}-\eta^{2}\right)^{1 / 2} Q_{o}\left(\xi_{o}\right)\right]=2 \epsilon_{o} /\left[a\left(\xi_{o}^{2}-\eta^{2}\right)^{1 / 2} \log \frac{\xi_{o}+1}{\xi_{o}-1}\right] .
$$

Solving now the same problem on the basis of (3), we first observe that with $\cos \theta^{\prime}=$ $u, f=f(u)$ is an even function of $u$ only, because of symmetry. In (3) $r^{\prime}$ is the distance from the origin of points $\xi_{o}, \eta$ on the spheroid and $d S^{\prime}=h_{\eta} h_{\phi} d \eta d \phi^{\prime}=a c\left(\xi_{o}^{2}-\eta^{2}\right)^{1 / 2} d \eta d \phi^{\prime}$. Substitution and integration with respect to $\phi^{\prime}$ simplify (3) into:

$$
\int_{-1}^{1} \frac{P_{n}(u)}{r^{\prime n+1}} f(u) a c\left(\xi_{o}^{2}-\eta^{2}\right)^{1 / 2} d \eta=2 \epsilon_{o} \delta_{n 0} ; \quad n=0,1,2, \cdots .
$$

Finally, the relations $\eta=(a / c) u /\left(\xi_{o}^{2}-u^{2}\right)^{1 / 2}, r^{\prime}=a \xi_{o} /\left(\xi_{o}^{2}-u^{2}\right)^{1 / 2}$ can be used to express all variables in the integrand in terms of $u$. The result is

$$
\begin{aligned}
\int_{-1}^{1} P_{n}(u)\left(1-u^{2} / \xi_{o}^{2}\right)^{(n-2) / 2}\left[\xi_{o}^{2}-\left(a^{2} / c^{2}\right) u^{2} /\left(\xi_{o}^{2}-u^{2}\right)\right]^{1 / 2} f(u) d u= & 2 \epsilon_{o} \delta_{n 0} \xi_{o} a^{n-1} ; \\
& n=0,1,2, \cdots .
\end{aligned}
$$

For odd values of $n$ this relation is satisfied automatically, $P_{n}(u)$ being an odd and the rest of the integrand an even function of $u$. For $n=2 m$ the equations are satisfied for all $m=0,1,2, \cdots$ if

$$
\left[\xi_{o}^{2}-\left(a^{2} / c^{2}\right) u^{2} /\left(\xi_{o}^{2}-u^{2}\right)\right]^{1 / 2} f(u)=A, \text { a constant. }
$$

Indeed, for $m=1,2, \cdots(7)$ becomes

$$
A \int_{-1}^{1}\left(1-u^{2} / \xi_{0}^{2}\right)^{m-1} P_{2 m}(u) d u=0,
$$

a relation that is obviously satisfied since $\left(1-u^{2} / \xi_{o}^{2}\right)^{m-1}$, being a polynomial of degree $2 m-2$, may be expanded into the finite sum $\sum_{i=0}^{2 m-2} a_{j} P_{i}(u)$, whereas $\int_{-1}^{1} P_{2 m}(u) P_{i}(u) d u$ $=0$ for $j \leq 2 m-2$. The remaining equation, for $m=0$, serves to evaluate $A$. Thus, with $P_{0}(u)=1$,

$$
A \int_{-1}^{1}\left(1-u^{2} / \xi_{o}^{2}\right)^{-1} d u=2 \epsilon_{o} \xi_{o} / a, \quad \text { or } \quad A=\left(2 \epsilon_{o} / a\right) / \log \frac{\xi_{o}+1}{\xi_{o}-1} .
$$

Substitution of this value in (8) yields for $f(u)$ a form identical to (5), if $\left(a^{2} / c^{2}\right) u^{2} /\left(\xi_{o}^{2}-\right.$ $\left.u^{2}\right)=\eta^{2}$ is also used. The uniqueness theorem in electrostatics suffices in establishing this solution as unique for the problem at hand. A direct and more desirable approach would be to establish uniqueness for the set of equations (7). It turns out to be a difficult task, however.

A similar method can be used to solve the oblate spheroid problem. Conversely, the corresponding sphere problem can be solved using spheroidal coordinates. Expansions of $1 / R$ in such coordinates are given in [3].

3. Possible generalizations. A general method starts from Eq. (3), which for axially symmetric shapes reduces to

$$
2 \epsilon_{o} \delta_{n 0}=\int_{-1}^{1} \frac{P_{n}(u)}{\left[r^{\prime}(u)\right]^{n+1}} f(u) h(u) d u, \quad n=0,1,2, \cdots,
$$


where $h(u)$ is a known function related to the shape of $S$. A possible approach is to use Schmidt's orthogonalization process to build an orthonormal set of functions $g_{n}(u)$ $(n=0,1, \cdots)$ over $-1 \leq u \leq 1$ on the basis of the set $P_{n}(u) w(u) /\left[r^{\prime}(u)\right]^{n+1}$, where $w(u)$ is a suitable weight function. Expansion of $f(u) h(u)$ in terms of the set $g_{\mathbf{x}}(u)$ leads to expansion coefficients that can be evaluated by means of diagonal matrices (explicitly). Diagonal and nearly diagonal matrices are also obtained for $g_{n}(u)$ in terms of $P_{n} w / r^{n+1}$. However, this general approach involves, among others, questions of convergence and completeness. The spheroid, treated previously, is a special case in which the expansion of $f(u) h(u)$ contains only one term, the first. For further details and, in particular, for related topics in electromagnetic scattering the reader is referred to [7] and the more complete and up-to-date set of papers cited therein.

\section{REFERENCES}

[1] O. D. Kellogg, Foundations of potential theory, Dover Publications, Inc., New York, N. Y., Chapter XI (1953)

[2] J. D. Jackson, Classical electrodynamics, John Wiley \& Sons, Inc., New York, N. Y., Chapter 1 (1962)

[3] P. M. Morse and H. Feshbach, Methods of theoretical physics, McGraw-Hill Book Company, Inc., New York, N. Y., pp. 1274 and 1284-96 (1953)

[4] W. R. Smythe, Charged right circular cylinder, J. Appl. Phys. 27, 917-920 (1956)

[5] W. R. Smythe, Charged sphere in cylinder, J. Appl. Phys. 31, 553-556 (1960)

[6] P. C. Waterman, Matrix formulation of electromagnetic scattering, Proc. IEEE 53, 805-812 (1965)

[7] P. C. Waterman, Symmetry, unitarity, and geometry in electromagnetic scattering, Phys. Rev. D3, 825-839 (1971)

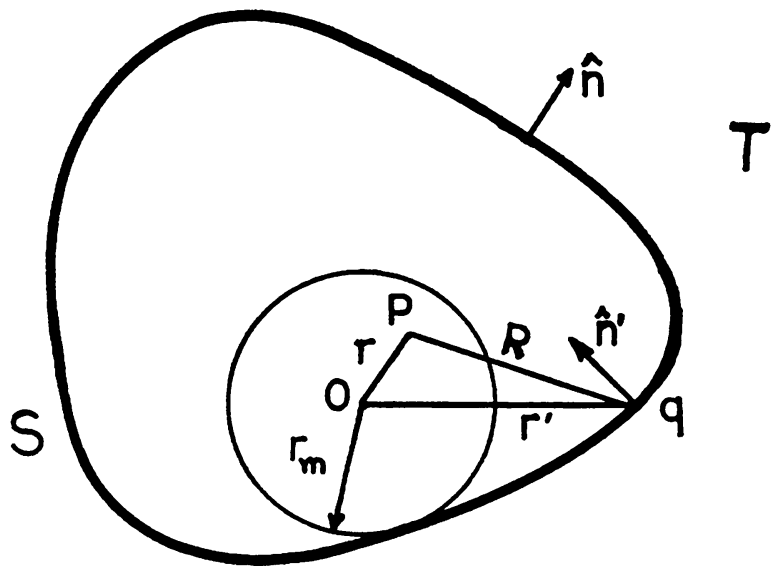

Fig. 1. The geometry of an arbitrary conductor. 